03,12

\title{
Влияние размерных эффектов на электронную структуру гексагонального теллурида галлия
}

\author{
(С) А.В. Кособуцкий ${ }^{1}$, С.Ю. Саркисов ${ }^{2}$ \\ ${ }^{1}$ Кемеровский государственный университет, \\ Кемерово, Россия \\ 2 Национальный исследовательский Томский государственный университет, \\ Томск, Россия \\ E-mail: kosobutsky@kemsu.ru
}

(Поступила в Редакцию 28 февраля 2018 г.)

С использованием методов теории функционала плотности выполнены расчеты электронной зонной структуры слоистого полупроводника GaTe гексагональной модификации. Структурные параметры объемного кристалла с симметрией $\beta$-политипа определены с учетом ван-дер-ваальсовых взаимодействий и согласуются с экспериментальными данными для поликристаллических пленок в пределах 2\%. Получены оценки положения экстремумов верхней валентной зоны и нижней зоны проводимости относительно уровня вакуума для объемного $\beta$-GaTe и для ультратонких пластин с числом элементарных слоев от 1 до 10, что соответствует диапазону толщины $0.5-8 \mathrm{~nm}$. Расчеты показывают, что гексагональный GaTe является непрямозонным полупроводником с шириной запрещенной зоны, варьирующейся от $0.8 \mathrm{eV}$ в объемном материале до $2.3 \mathrm{eV}$ в монослое.

Работа поддержана грантом № 8.2.18.2017 в рамках Программы повышения конкурентоспособности ТГУ.

DOI: $10.21883 /$ FTT.2018.09.46378.052

\section{1. Введение}

Теллурид галлия (GaTe) относится к семейству слоистых кристаллов $\mathrm{A}^{\mathrm{III}} \mathrm{B}^{\mathrm{VI}}$, перспективных для использования в нелинейной оптике и оптоэлектронике. В отличие от слоистых сульфида и селенида галлия, имеющих гексагональную симметрию (наиболее общими политипами являются $\beta$-GaS и $\varepsilon$-GaSe с идентичными по своей структуре слоями), объемные кристаллы теллурида галлия кристаллизуются преимущественно в моноклинной сингонии ( $m$-GaTe с пространственной группой $B 2 / m)$. По своим электронным свойствам соединение $m$-GaTe является прямозонным полупроводником с шириной запрещенной зоны около $1.7 \mathrm{eV}$ при комнатной температуре [1]. Намеренно нелегированные образцы GaTe, a также GaSe и их твердые растворы как правило демонстрируют проводимость $p$-типа [2], что можно связать с положением уровня зарядовой нейтральности в нижней половине запрещенной щели $[3,4]$.

Выполненные в последние годы исследования нанослоев GaTe показали их высокую фоточувствительность и перспективность использования в качестве компонентов квазидвумерных (2D) ван-дер-ваальсовых гетероструктур [5,6], в которых различные по своему составу $2 \mathrm{D}$ слои располагаются друг над другом в требуемой последовательности с целью управления физическими свойствами и повышения производительности электронных устройств [7].

Возможность получения тонких пленок GaTe в гексагональной фазе ( $\beta$-GaTe) была впервые показана в [8], где был также обнаружен переход $\beta$-GaTe $\rightarrow m$-GaTe при отжиге образца, позднее наблюдавшийся в [9]. В работе [10] методом вертикальной зонной плавки под высоким давлением инертного газа были выращены крупные монокристаллы $\beta$-GaTe и дана оценка их механических и оптических свойств. В работе [11] показано, что ультратонкие пластинки GaTe с числом слоев от 1 до 3, полученные методом микромеханического расслоения объемного кристалла $m$-GaTe, испытывают спонтанный переход в гексагональную фазу. Использование подложки GaAs (001) позволило в [12] вырастить методом молекулярно-пучковой эпитаксии гексагональные слои GaTe толщиной до $\sim 90 \mathrm{~nm}$.

Опубликованные в литературе теоретические исследования гексагонального GaTe преимущественно фокусируются на рассмотрении характеристик единичного тетраслоя этого соединения (например, [13]). Целью настоящей работы является изучение влияния квантоворазмерных эффектов на электронные свойства $\beta$-GaTe c различным числом элементарных слоев.

\section{2. Метод расчета}

Расчеты электронной структуры выполнялись в рамках теории функционала плотности (DFT) с использованием приближения проектированных присоединенных волн для учета электрон-ионного взаимодействия. Электронные волновые функции раскладывались в ряд по плоским волнам с максимальной энергией $50 \mathrm{Ry}$. Интегрирование по зоне Бриллюэна проводилось на сетках $12 \times 12 \times 2$ для объемного кристалла и $12 \times 12 \times 1$ для нанослоев. Моделирование квазидвумерных структур в 
рамках подхода с использованием периодических граничных условий подразумевает использование суперьячеек, включающих пластину кристалла и вакуумный слой достаточной толщины, который вводится для устранения взаимодействий между трансляционно повторяющимися элементами. В настоящей работе использовались суперъячейки с количеством атомов от 4 до 40 и толщиной вакуумного промежутка не менее $15 \AA$.

Результаты расчетов структурных параметров слоистых кристаллов $\mathrm{A}^{\mathrm{III}} \mathrm{B}^{\mathrm{VI}}$ существенно зависят от выбора обменно-корреляционного функционала [14]. Для получения более близких к эксперименту результатов требуется учитывать влияние сил Ван-дер-Ваальса, играющих важную роль в слоистых материалах. Функционал vdW-DF2-C09 показал высокую производительность в предыдущих исследованиях соединений $\mathrm{A}^{\mathrm{III}} \mathrm{B}^{\mathrm{VI}}[3,14,15]$ и поэтому был выбран для расчетов параметров решетки $\beta$-GaTe в данной работе. Процесс оптимизации структуры останавливался, когда действующие на атомы силы понижались до значений менее $0.01 \mathrm{eV} / \AA ̊$. Все численные расчеты проводились в программном пакете Quantum ESPRESSO [16].

\section{3. Результаты и обсуждение}

3.1. Кристаллическая структура гексагональн о го GaTe. Слоистые полупроводники семейства $\mathrm{A}^{\mathrm{III}} \mathrm{B}^{\mathrm{VI}}$, такие как $\mathrm{GaS}, \mathrm{GaSe}$ и InSe, демонстрируют многообразие кристаллических модификаций, обусловленное возможностью различного расположения элементарных слоев относительно друг друга. При этом все слои имеют одинаковое внутреннее устройство, характеризуются гексагональной симметрией, и представляют собой пакеты из четырех параллельных атомных плоскостей, в которых атомы VI и III групп чередуются в последовательности VI-III-III-VI. Кристаллическая структура объемного $\beta$-GaTe показана на рис. 1 . Симметрия $\beta$-политипа описывается центросимметричной

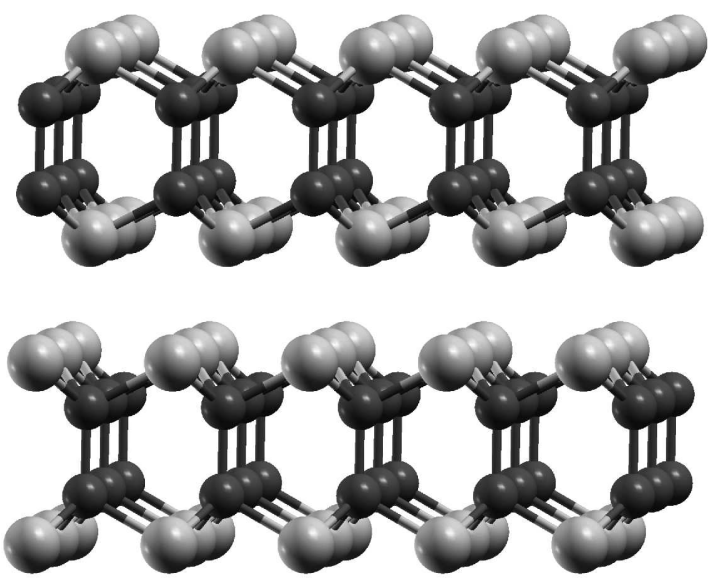

Рис. 1. Структура и расположение слоев в $\beta$-GaTe. Атомы металла обозначены шарами меньшего размера.
Таблица 1. Постоянные решетки $a$ и $c$, толщина элементарного слоя $d_{\text {intra }}$ и межслоевое расстояние $d_{\text {inter }}$ кристалла $\beta$-GaTe $(\AA)$

\begin{tabular}{l|l|c|c|c}
\hline \multicolumn{1}{c|}{ Метод } & $a$ & $c$ & $d_{\text {intra }}$ & $d_{\text {inter }}$ \\
\hline Теория & 4.06 & 16.63 & 4.97 & 3.35 \\
Эксперимент [8,11] & 4.06 & 16.96 & & \\
Эксперимент [9] & 4.1 & 16.38 & &
\end{tabular}

пространственной группой $P 6_{3} / m m c$, в элементарной ячейке находятся 8 атомов из двух соседних слоев. Каждый атом теллура связан с тремя соседними атомами галлия, тогда как каждый атом галлия находится в тетраэдрическом окружении из трех атомов теллура и одного атома галлия. Отметим, что структура моноклинного GaTe является более сложной по сравнению с гексагональным кристаллом: в отличие от гексагональной модификации, в слоях $m$-GaTe присутствуют связи $\mathrm{Ga}-\mathrm{Ga}$, ориентированные как перпендикулярно слоям, так и вдоль слоев.

В табл. 1 приведены оптимизированные с использованием ван-дер-ваальсова функционала vdW-DF2-C09 постоянные решетки $a$ и $c$ соединения $\beta$-GaTe в сопоставлении с экспериментальными данными $[8,9,11]$. Теоретические данные согласуются с результатами измерений для поликристаллических образцов в пределах 2\%. Сравнение рассчитанных межатомных расстояний в $\mathrm{GaSe}$ [14] и GaTe показывает, что длина связи $\mathrm{Ga}-\mathrm{Ga}$ почти не меняется при замене атома $\mathrm{Se}$ на Те $(2.43$ и $2.42 \AA$ соответственно), тогда как длина катион-анионной связи возрастает с 2.47 до $2.67 \AA$ вследствие большего радиуса атома Те $(1.37 \AA)$ по сравнению с $\operatorname{Se}(1.18 \AA)$. Это приводит к росту постоянных решетки и толщины элементарного слоя $d_{\text {intra }}$ в $\beta$-GaTe на $4-8 \%$. Интересно, что при переходе от $\varepsilon$-GaSe к $\beta$-GaTe ширина ван-дерваальсовой щели $d_{\text {inter }}$ также возрастает на $\sim 5 \%$, тогда как в $\beta$-GaS и $\varepsilon$-GaSe величины $d_{\text {inter }}$ являются довольно близкими [3].

3.2. Электронные свойства. Структурные параметры, полученные на этапе оптимизации решетки с использованием функционала vdW-DF2-C09, использовались при расчетах электронных зонных спектров объемного кристалла $\beta$-GaTe и нанослойных структур с числом элементарных слоев $n=1-10$. При моделировании нанослоев брались структурные параметры объемного $\beta$-GaTe, поскольку слабые межслоевые взаимодействия практически не влияют на строение отдельного слоя. Результаты расчетов представлены на рис. 2. Для удобства сопоставления зонных спектров на рис. $2, a-d$ отсчет энергий ведется от энергии последнего занятого уровня. Вычисления проводились в рамках стандартного приближения PBE-GGA, так как полученная с использованием ван-дер-ваальсового функционала дисперсионная зависимость $E(k)$ не показывает принципиальных отличий [14]. 

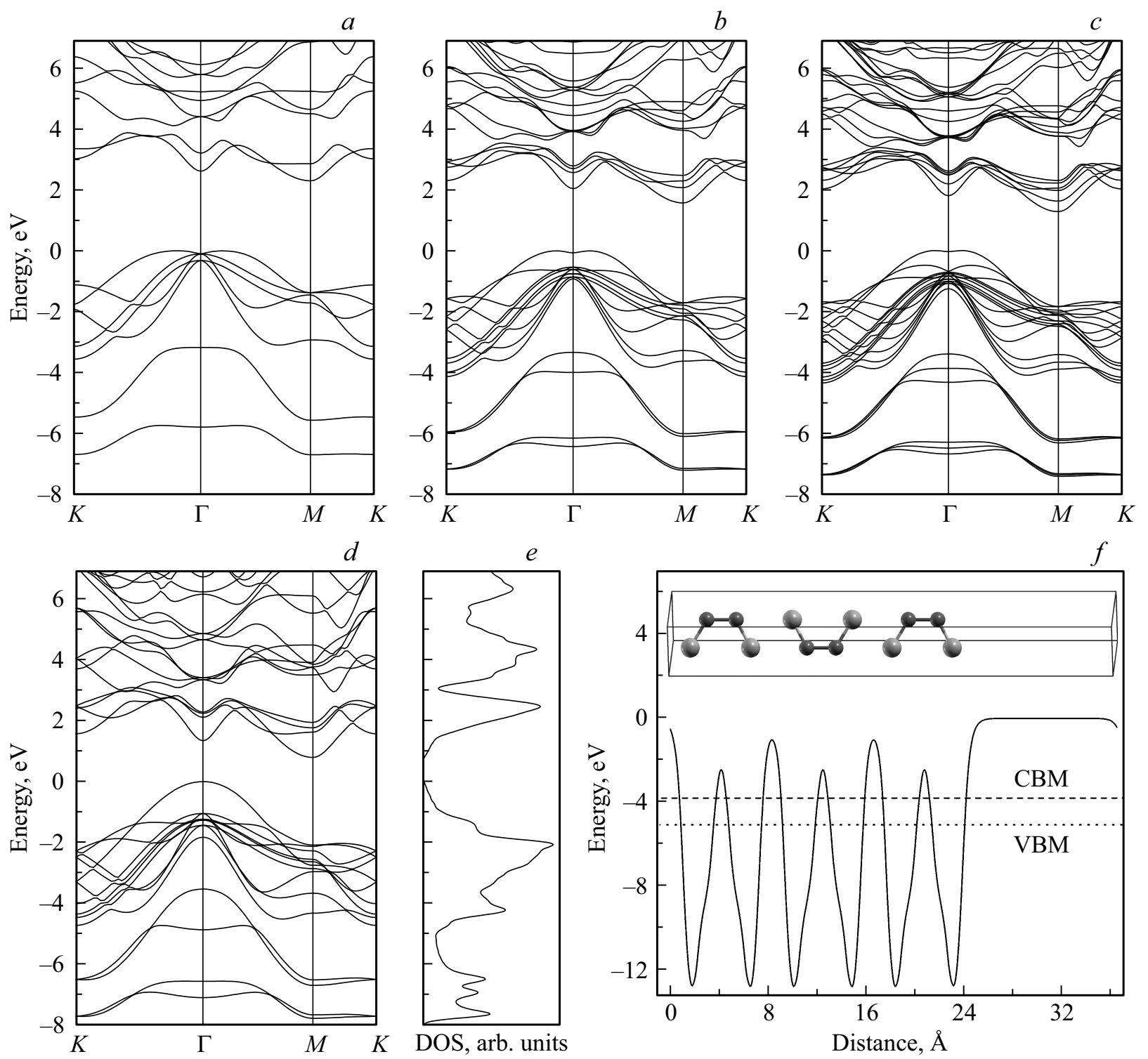

Рис. 2. Зонные структуры пластинок гексагонального GaTe с числом слоев $n$ от 1 до 3 соответственно $(a-c)$, зонный спектр и плотность состояний объемного $\beta$-GaTe $(d, e)$ и усредненный в плоскости $(001)$ электростатический потенциал $\mathrm{GaTe}$ при $n=3(f)$ вдоль оси $c$ ячейки, показанной на вставке.

Хорошо известно, что расчеты в рамках DFT приводят к заниженным значениям ширины запрещенной зоны $E_{g}$ полупроводников и изоляторов. Так, наши предыдущие расчеты для моноклинного $m$-GaTe в [3] дали $E_{g}$ около $0.94 \mathrm{eV}$, тогда как типичным результатом измерений спектров фотолюминесценции и оптического поглощения для $m$-GaTe при комнатной температуре является $E_{g}=1.65-1.66 \mathrm{eV}[1,5]$. С целью получения более близких значений $E_{g}$ к результатам измерений оптической ширины запрещенной зоны, в настоящей работе использовалась систематическая поправка $0.72 \mathrm{eV}$ к энергиям межзонных переходов нанослоев $\mathrm{GaTe}$ из расчетов в рамках DFT. Стоит отметить, что аналогичный способ коррекции величин межзонных переходов использовался в [17] для InSe и позволил получить очень хорошее согласие с экспериментом при описании оптических свойств нанослоев селенида индия различной толщины.

В пренебрежении спин-орбитальными эффектами зонные спектры монослоя и объемного кристалла $\beta$-GaTe содержат соответственно 7 и 14 ветвей в интервале энергий от -8 до $0 \mathrm{eV}$, соответствующем занятым состояниям. Анализ парциальных вкладов в полную плотность состояний $\beta$-GaTe показывает, что состояния в диапазоне от -13 до $-11 \mathrm{eV}$ имеют Те $5 s$-характер. Энергетические уровни в диапазоне от -5 до $0 \mathrm{eV}$ сформированы путем гибридизации $p$-орбиталей галлия и теллура, с преимущественным вкладом последних. Состояния в окрестности вершины валентной зоны образованы из $5 p_{z}$-орбиталей Те, которые ориентированы вдоль оси $c$ гексагонального политипа и расположены вдоль 
границ слоев. Нижние зоны проводимости (от $\sim 0.8$ до $3 \mathrm{eV}$ ) имеют преимущественные вклады $5 p$-орбиталей Te и $4 s-, 4 p$-орбиталей Ga. $4 s$-орбитали галлия также имеют значительный вклад в интервале от -8 до $-5 \mathrm{eV}$, где расположены связывающие и антисвязывающие состояния димера $\mathrm{Ga}-\mathrm{Ga}$.

Зонная структура монослойного GaTe имеет типичную форму для соединений $\mathrm{A}^{\mathrm{III}} \mathrm{B}^{\mathrm{VI}}[13,18]$. Вершина валентной зоны находится на линии $\Gamma-K$ двумерной зоны Бриллюэна, при этом на линии $\Gamma-M$ присутствует близкий по энергии локальный максимум, обеспечивающий дисперсионной зависимости в окрестности т. Г симметричный вид (рис. 2,a). В отличие от $\mathrm{GaS}$ и $\mathrm{GaSe}[18]$, в монослое GaTe локальный минимум верхней валентной зоны в т. Г лежит очень близко к более глубоким уровням, обусловленным $p_{x}$ - и $p_{y}$-орбиталями аниона. Переход от монослоя к структурам с большим количеством слоев сопровождается расщеплением энергетических зон, постепенным приближением максимума валентной зоны к т. Г и сдвигом вниз находящегося в т. $M$ дна зоны проводимости (рис. $2, a-d$ ).

В табл. 2 представлены рассчитанные значения ширины непрямой и прямой (переход $\Gamma_{v}-\Gamma_{c}$ ) запрещенной щели гексагонального GaTe в зависимости от числа $n$ элементарных слоев, а также энергетическое положение центра запрещенной щели $\left(E_{\mathrm{BGC}}\right)$, максимума валентной зоны $\left(E_{\mathrm{VBM}}\right)$ и минимума нижней зоны проводимости $\left(E_{\mathrm{CBM}}\right)$ относительно уровня электрона в вакууме. Энергии $E_{\mathrm{VBM}}$ и $E_{\mathrm{CBM}}$ нанослоев определялись согласно выражениям

$$
\begin{aligned}
& E_{\mathrm{VBM}}=\left(\varepsilon_{\mathrm{VBM}}-\Delta\right)-V_{\mathrm{vac}}, \\
& E_{\mathrm{CBM}}=\left(\varepsilon_{\mathrm{CBM}}+\Delta\right)-V_{\mathrm{vac}},
\end{aligned}
$$

где $\varepsilon_{\mathrm{VBM}}, \varepsilon_{\mathrm{CBM}}-$ энергетические уровни, определенные из решения уравнений Кона-Шэма, $V_{\text {vac }}$ - электростатический потенциал в центре вакуумного слоя, $\Delta=0.36 \mathrm{eV}$ - поправка, устраняющая занижение $E_{g}$ как описано выше. Результаты расчетов электростатического потенциала на примере трехслойного $\beta$-GaTe показаны на рис. 2, $f$.

Стоит отметить, что использованный в (1), (2) способ коррекции валентных зон и зон проводимости не затрагивает положение центра запрещенной щели относительно уровня вакуума, который в точной DFT имеет конкретный физический смысл: $E_{\mathrm{BGC}}=-(I+A) / 2$, где $I$ - потенциал ионизации, $A$ - электронное сродство [19]. Сопоставление полученных результатов для монослоя GaTe с данными расчетов [13] с использованием ресурсозатратного метода гибридного функционала (табл. 2) показывает хорошее согласие - отличие наших расчетов от вычислений [13] не превышает $0.12 \mathrm{eV}$. Это говорит о переносимости введенной в (1), (2) поправки для энергий зонной структуры на слои GaTe различной толщины, что позволяет получать оценки для нанослоев с большим числом $n$, где применение более сложных методов расчетов чрезвычайно затруднительно.
Таблица 2. Параметры зонной структуры $\beta$-GaTe в зависимости от числа слоев $n\left(E_{g, i}-\right.$ непрямая ширина запрещенной зоны, $E_{g, d}-$ прямая ширина в т. $\Gamma ; E_{\mathrm{BGC}}, E_{\mathrm{VBM}}, E_{\mathrm{CBM}}-$ положение центра запрещенной щели и экстремумов валентной зоны и зоны проводимости (в $\mathrm{eV})$. Для монослойного $\mathrm{GaTe}$ $(n=1)$ в скобках приведены результаты расчетов с гибридным функционалом HSE06 [13])

\begin{tabular}{c|c|c|c|c|c}
\hline$n$ & $E_{g, i}$ & $E_{g, d}(\Gamma)$ & $E_{\mathrm{BGC}}$ & $E_{\mathrm{VBM}}$ & $E_{\mathrm{CBM}}$ \\
\hline 1 & $\begin{array}{c}2.30 \\
(2.22)\end{array}$ & $\begin{array}{c}2.71 \\
(\sim 2.63)\end{array}$ & $\begin{array}{c}-4.56 \\
(-4.64)\end{array}$ & $\begin{array}{c}-5.71 \\
(-5.75)\end{array}$ & $\begin{array}{c}-3.41 \\
(-3.53)\end{array}$ \\
\hline 2 & 1.58 & 2.11 & -4.48 & -5.27 & -3.69 \\
\hline 3 & 1.28 & 1.84 & -4.44 & -5.08 & -3.80 \\
\hline 4 & 1.13 & 1.69 & -4.42 & -4.98 & -3.85 \\
\hline 5 & 1.04 & 1.60 & -4.40 & -4.92 & -3.88 \\
\hline 10 & 0.88 & 1.44 & -4.36 & -4.80 & -3.92 \\
\hline $\begin{array}{c}\text { Объемный } \\
\text { материал }\end{array}$ & 0.79 & 1.34 & -4.33 & -4.73 & -3.94
\end{tabular}

Как видно из табл. 2, с ростом $n$ уровни $E_{\mathrm{VBM}}$ и $E_{\mathrm{CBM}}$ смещаются соответственно вверх и вниз по энергетической шкале, что приводит к уменьшению ширины запрещенной зоны на $1.51 \mathrm{eV}$ при переходе от изолированного монослоя к объемному материалу. Возрастание $E_{\mathrm{VBM}}$ означает понижение потенциала ионизации при увеличении толщины кристаллической пластинки $\beta$-GaTe. Согласно полученным нами данным, $\Delta I=-0.79 \mathrm{eV}$ при увеличении числа слоев от 1 до 5 . Близкие результаты демонстрируют также расчеты для гексагонального сульфида галлия в [20]. Напротив, выполненные в [21] расчеты для моноклинной модификации $\mathrm{GaTe}$ не показывают заметной зависимости $E_{\mathrm{VBM}}$ от числа слоев и дают $I=5.09-5.21 \mathrm{eV}$ при $n=1-5$. В соответствии с оценками [21], изменение $E_{g}$ соединения $m$-GaTe относительно мало (в пределах $0.42 \mathrm{eV}$ в интервале $n=1-5)$ и происходит преимущественно за счет изменения $E_{\mathrm{CBM}}$. Таким образом, гексагональная модификация предоставляет больше возможностей по управлению шириной запрещенной щели.

Согласно полученным данным, величина $E_{g}$ объемного $\beta$-GaTe составляет около $0.8 \mathrm{eV}$, тогда как для слоистых сульфида и селенида галлия эксперимент дает соответственно $2.5 \mathrm{eV}$ и $2.0 \mathrm{eV}(T=300 \mathrm{~K})$ [3]. Уменьшение $E_{g}$ в ряду соединений $\mathrm{Ga} X(X=\mathrm{S}, \mathrm{Se}, \mathrm{Te})$ с увеличением массы аниона обусловлено понижением степени ионности связи $\mathrm{Ga}-X$. Следует отметить, что представленные в литературе экспериментальные данные для $E_{g}$ гексагонального GaTe демонстрируют большой разброс. Так, из оптических спектров пропускания поликристаллических пленок $\beta$-GaTe в [9] определено значение ширины запрещенной щели в диапазоне $1.75-1.9 \mathrm{eV}$, тогда как для пластинок теллурида галлия, отщепленных от 
слитка $\beta$-GaTe, в $[10]$ получено $E_{g}=1.65 \mathrm{eV}$. Последнее значение совпадает с измерениями для $m$-GaTe, что может говорить о присутствии моноклинной фазы в образце.

Фотолюминесцентные исследования гексагональных кристаллов $\mathrm{GaSe}_{x} \mathrm{Te}_{1-x}$, выращенных на подложке $\mathrm{GaSe}$, в [22] обнаружили эмиссионный пик при $1.44 \mathrm{eV}$ в спектре разбавленных твердых растворов $(x \leq 0.05)$. Этот результат неплохо согласуется с рассчитанным нами значением ширины прямой запрещенной щели $1.34 \mathrm{eV}$ объемного $\beta$-GaTe. Приведенные в [22] теоретические оценки с обменным потенциалом mBJ (ТВ09), который в значительной степени компенсирует ошибку локального приближения DFT при описании положения зон проводимости полупроводников [23,24], также подтверждают понижение $E_{g}$ в $\beta$-GaTe по сравнению с $m$-GaTe.

\section{4. Заключение}

В представленной работе выполнены расчеты параметров зонной структуры слоистых кристаллов теллурида галлия гексагональной модификации. Рассмотрена кристаллическая упаковка $\beta$-политипа с числом элементарных слоев от 1 до 10. Получены оценки энергетического положения краев валентной зоны и зоны проводимости относительно уровня вакуума. Результаты показывают непрямозонный характер запрещенной щели всех рассмотренных нанослоев и объемного кристалла. Энергия непрямого перехода принимает наибольшее значение в монослое $\left(E_{g, i}=2.3 \mathrm{eV}\right)$ и уменьшается при добавлении новых слоев вследствие сдвига вверх максимума валентной зоны и одновременного понижения минимума зоны проводимости. Рассчитанные энергии непрямого и прямого переходов для объемного $\beta$-GaTe соответственно составляют 0.79 и $1.34 \mathrm{eV}$. Полученные данные показывают, что гексагональная модификация дает больше возможностей по управлению шириной запрещенной щели по сравнению с моноклинным GaTe.

\section{Список литературы}

[1] J.F. Sánchez-Royo, A. Segura, V. Muñoz. Phys. Status Solidi A 151, 257 (1995)

[2] Б.Г. Тагиев, О.Б. Тагиев. ФТТ 59, 1060 (2017).

[3] V.N. Brudnyi, S.Yu. Sarkisov, A.V. Kosobutsky. Semicond. Sci. Technol. 30, 115019 (2015).

[4] A.V. Kosobutsky, S.Yu. Sarkisov, V.N. Brudnyi. J. Phys. Chem. Solids 74, 1240 (2013).

[5] P. Hu, J. Zhang, M. Yoon, X.-F. Qiao, X. Zhang, W. Feng, P. Tan, W. Zheng, J. Liu, X. Wang, J.C. Idrobo, D.B. Geohegan, K. Xiao. Nano Res. 7, 694 (2014).

[6] F. Wang, Z. Wang, K. Xu, F. Wang, Q. Wang, Y. Huang, L. Yin, J. He. Nano Lett. 15, 7558 (2015).

[7] И.В. Антонова. ФТП 50, 67 (2016).

[8] С.А. Семилетов, В.А. Власов. Кристаллография 8, 877 (1963). [S.A. Semiletov, V.A. Vlasov. Sov. Phys.-Crystallogr. 8, 704 (1964)].
[9] E.G. Gillan, A.R. Barron. Chem. Mater. 9, 3037 (1997).

[10] N.N. Kolesnikov, E.B. Borisenko, D.N. Borisenko, A.V. Timonina. J. Cryst. Growth 365, 59 (2013).

[11] Q. Zhao, T. Wang, Y. Miao, F. Ma, Y. Xie, X. Ma, Y. Gu, J. Li, J. He, B. Chen, S. Xi, L. Xu, H. Zhen, Z. Yin, J. Li, J. Rena, W. Jie. Phys. Chem. Chem. Phys. 18, 18719 (2016).

[12] C.J. Bae, J. McMahon, H. Detz, G. Strasser, J. Park, E. Einarsson, D.B. Eason. AIP Adv. 7, 035113 (2017).

[13] H.L. Zhuang, R.G. Hennig. Chem. Mater. 25, 3232 (2013).

[14] S.Y. Sarkisov, A.V. Kosobutsky, S.D. Shandakov. J. Solid State Chem. 232, 67 (2015).

[15] С.Ю. Саркисов, А.В. Кособуцкий, В.Н. Брудный, Ю.Н. Журавлев. ФТТ 57, 1693 (2015).

[16] P. Giannozzi, S. Baroni, N. Bonini. J. Phys.: Condens. Matter 21, 395502 (2009).

[17] D.A. Bandurin, A.V. Tyurnina, G.L. Yu, A. Mishchenko, V. Zólyomi, S.V. Morozov, R.K. Kumar, R.V. Gorbachev, Z.R. Kudrynskyi, S. Pezzini, Z.D. Kovalyuk, U. Zeitler, K.S. Novoselov, A. Patané, L. Eaves, I.V. Grigorieva, V.I. Fal'ko, A.K. Geim, Y. Cao. Nature Nanotechnol. 12, 223 (2017).

[18] D.V. Rybkovskiy, A.V. Osadchy, E.D. Obraztsova. Phys. Rev. B 90, 235302 (2014).

[19] J.P. Perdew, M. Levy. Phys. Rev. Lett. 51, 1884 (1983).

[20] 3.А. Джахангирли, Ф.М. Гашимзаде, Д.А. Гусейнова, Б.Г. Мехтиев, Н.Б. Мустафаев. ФТТ 58, 1707 (2016).

[21] J.A. Olmos-Asar, C.R. Leão, A. Fazzio. RSC Adv. 7, 32383 (2017).

[22] J.J. Fonseca Vega. Bandgap engineering of gallium telluride. PhD Thesis. University of California, Berkeley (2017). P. 53.

[23] А.В. Кособуцкий, А.Б. Гордиенко. ФТТ 57, 1922 (2015).

[24] A.V. Kosobutsky, Yu.M. Basalaev. Solid State Commun. 199, 17 (2014).

Редактор Т.Н. Василевская 